\title{
BCCN4
}

\section{MICROMECHANICAL MODELS FOR TRANSVERSAL ELASTIC MODULUS IN UNIDIRECTIONAL LAMINATES}

\author{
Lucas L. Vignoli $^{(1,2)}$, Marcelo M. Savi ${ }^{(1)}$
}

(1) Center for Nonlinear Mechanics, COPPE, Department of Mechanical Engineering, Universidade Federal do Rio de Janeiro, Brazil

(2) Department of Mechanical Engineering, Universidade Federal do Rio de Janeiro - Campus Macaé, Brazil

https://doi.org/10.21452/bccm4.2018.03.02

\begin{abstract}
This paper deals with the first step required for composite design: the equivalent macromechanical properties according to the constituents. Assuming a transversally isotropic lamina, the material has five independent properties, namely longitudinal and transversal elastic modulus, in-plane and out-of-plane shear modulus and in-plane Poisson ratio. The longitudinal elastic modulus has a very reasonable prediction by the well-known Rule of Mixtures. On the other hand, the transversal elastic modulus estimative by the same model is poor compared with experimental data. There exist many additional proposed models, therefore all of them are based in hypotheses that usually are not true for real composites, as unit cell symmetry. Due to this fact, the predictions of the following nine models are evaluated: Asymptotic Homogenization with hexagonal unit cell, Asymptotic Homogenization with square unit cell, Bridging, Chamis, Generalized Self Consistent, Halpin-Tsai, modified Halpin-Tsai, Mori-Tanaka and Rule of Mixture. To stablish a comparison, a set of 65 experimental data are compiled from the literature. A semi-empirical modification of the Rule of Mixture is proposed based in a statistical adjust of the experimental data.
\end{abstract}

\section{INTRODUCTION}

The capability to estimate macromechancal properties of a unidirectional composite using the constituent data is a fundamental tool for composite design advance. Despite the recent advance of failure modelling due to the World Wide Failure Exercise [1-3], there is still a considerable lack of knowledge about the estimation of different micromechanical models, mainly for transversal elastic properties and strengths. The present paper is intended to provide a comparison between 10 models, 5 semi-empirical and 5 elasticity-based, and 65 experimental data for the transversal elastic modulus $\left(E_{2}\right)$. 
It is evident that the elasticity-based models have a physical basis much more consistent than the semi-empirical ones. Nevertheless, the goal of this comparative study is to evaluate which models, and consequently, which assumptions are more representative of the real structure. Figure 1 shows a microscopy image of a unidirectional lamina. The fiber distribution indicates an asymmetric pattern. Additionally, if a square unit cell is defined, the fiber volume fraction is dependent of where this square is located. Hence, the adjustable factors of semi-empirical model may represent a reasonable approximation for general laminae.

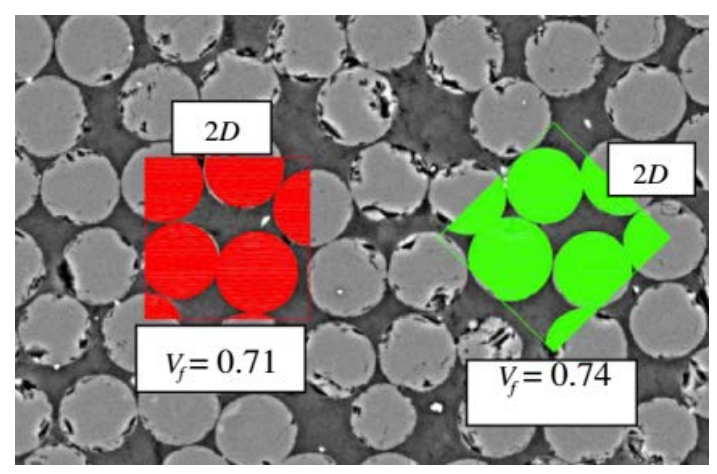

Figure 1: Microscopy of a real unidirectional lamina without simmetric arrangement [4].

\section{ANALYTICAL MODELS}

The analytical models presented in the literature are usually separated in two different approaches: semi-empirical and elasticity-based. In this study, the Rule of Misture, Chamis and Halpin-Tsai are semi-empirical, while Asymptotic Homogenization, Bridging, Generalized Self Consistent and Mori-Tanaka are elasticity-based. For simplicity, just the equations for semi-empirical models are completely defined and a brief explanation about the main idea of the elasticity-based models is presented.

The Rule of Mixture (ROM) is the simplest model and it is based on the idea of considering fibers and matrix as springs in series or in parallel, according to the applied load. For the longitudinal elastic modulus, this assumption works very well, since there is not abrupt perturbation on the stress and strain fields when a longitudinal load is applied (on the elastic range), and the lamina behaviour is similar to springs in parallel. For transversal load, the stress and strain fields are highly perturbed by the fibers distribution. Therefore, even with this limitation in mind, transversal elastic modulus is estimated using a simplified 2D geometry as [5]

$$
E_{2}=\frac{E_{2}^{f} E^{m}}{E_{2}^{f}\left(1-V_{f}\right)+E^{m} V_{f}}
$$

where $E^{m}$ is the matrix elastic modulus, $E_{2}^{f}$ is the fiber transversal elastic modulus and $V_{f}$ is the fiber volume fraction.

The Chamis model (Ch) [6] proposes consider the nonlinear effect of the fiber and void volume fraction replacing $V_{f}$ by its square root $\sqrt{\left(1-V_{v}\right) V_{f}}$. Hence, the Chamis model uses the following equation 


$$
E_{2}=\frac{E^{m}}{1-\sqrt{\left(1-V_{v}\right) V_{f}}\left[1-\left(E^{m} / E_{2}^{f}\right)\right]}
$$

For this study, it is considered two different void contents: $V_{v}=0$ and $V_{v}=2.5 \%$.

The Halpin-Tsai model (HT) [7] includes an adjustable parameter, $\zeta_{E_{2}}$, fitted according to experimental data. The transversal elastic model is estimated by

$E_{2}=E^{m}\left(\frac{1+\zeta_{E_{2}} \eta_{E_{2}} V_{f}}{1-\eta_{E_{2}} V_{f}}\right)$

where

$\eta_{E_{2}}=\frac{\left(E_{2}^{f} / E^{m}\right)-1}{\left(E_{2}^{f} / E^{m}\right)+\zeta_{E_{2}}}$

Halpin \& Kardos [8] suggest that in absence of experimental data for proper calibration, this parameter may be computed by

$$
\zeta_{E_{2}}=2+40 V_{f}^{10}
$$

Recently, Ginet et al.[9] carried out a numerical study with nonperiodic fiber distribution and callibrated this parameter using the following equation

$$
\zeta_{E_{2}}=\left\{\begin{array}{ccc}
4.924-35.888 V_{f}+125.118 V_{f}^{2}-145.12 V_{f}^{3} & \text { if } & V_{f}<0.3 \\
1.5+5500 V_{f}^{18} & \text { if } \quad V_{f} \geq 0.3
\end{array}\right.
$$

In the present study, the use of Halpin-Tsai model with Eq.(6) is named modified HalpinTsai (HTm).

The proposed semi-empirical model is based in a modification of the Rule of Mixture and on the Halpin-Tsai idea to add a calibration parameter. The following equation is proposed

$$
E_{2}=E^{m}\left(\frac{1}{1+\xi_{E_{2}}\left[\left(E^{m} / E_{2}^{f}\right)-1 V_{f}\right.}\right)
$$

where $\xi_{E_{2}}$ is the calibrated term. Note that $\xi_{E_{2}}=1$ reproduces the Rule of Mixture. Unlike the Halpin-Tsai model, which consider just the influence of $V_{f}$ on the calibration, the present model also consider the ratio $E^{m} / E_{2}^{f}$. Using the Levenberg-Marquardt [10] to calibrate this term, it is suggest using

$$
\xi_{E_{2}}=\left[0.6897+0.4185 V_{f}-0.0036\left(E_{2}^{f} / E^{m}\right)\right]^{-2}
$$

The main ideas of the elasticity-based models are: 
(i) Asymptotic Homogenization (AH): using an asymptotic expansion of the displacement field and satisfy the boundary condition, according to the unit cell geometry, square (Ahs) [11] or hexagonal (Ahs) [12]; despite the need of infinity series to compute the proprieties, just the first two terms are necessary to convergence [13].

(ii) Bridging $(\mathrm{Br})$ : there exist two different approaches, one is based on the Generalized Self Consistent and the other on the Mori-Tanaka, therefore its main advance is the capability to implement nonlinear effects, like damage, using the bridging tensor [14].

(iii) Generalized Self Consistent (GSCM): this model assumes that fiber and matrix are approximately represented by concentric cylinder; by this hypothesis, a geometrically compatible displacement is proposed for each load and at last the average stress and strain fields are computed [15].

(iv) Mori-Tanaka (MT): the key point of this model is the eigenstrain concept and the Eshelby inclusion theory [16] and the definition of forth order tensors to relate the strain fields on the constituents [17].

\section{RESULTS AND DISCUSSION}

A set of 65 experimental data of $E_{2}$ was compiled from the literature [18-32] for the following comparison. Two different approaches are used for evaluation: the average error considering all the data available and the ranges of error, defining smaller than $10 \%$, between $10 \%$ and $20 \%$; between $20 \%$ and $30 \%$, between $30 \%$ and $40 \%$, between $40 \%$ and $50 \%$ and higher than $50 \%$. The first one gives a value easier to compare, while the second offer a useful measure which is able to check if the average error is representative or not. For instance, if one data has a discrepant error, the average value is highly influenced, while the ranges allow a better representation. The results with both methodologies are presented in Figure 2 and Figure 3. The following conclusions are highlighted from these figures:

i) The semi-empirical model, namely est(ROM) on the graphics, is the unique model able to estimate more than $50 \%$ of the cases with an error smaller than $10 \%$ and to obtain an average error smaller than $15 \%$.

ii) The void content does not result in a representative alteration on the Chamis model prediction.

iii) The modified Halpin-Tsai obtained a considerable amount of cases with errors higher than 50\%, increasing the average error. Comparing with the classical Halpin-Tsai model, there is no advantage in this new equation for $\zeta_{E_{2}}$.

iv) All the elasticity-based models had a similar prediction, but the semi-empirical models (except the Rule of Mixture) obtained a smaller error. This result indicates the capability of application of this simplified approaches. 


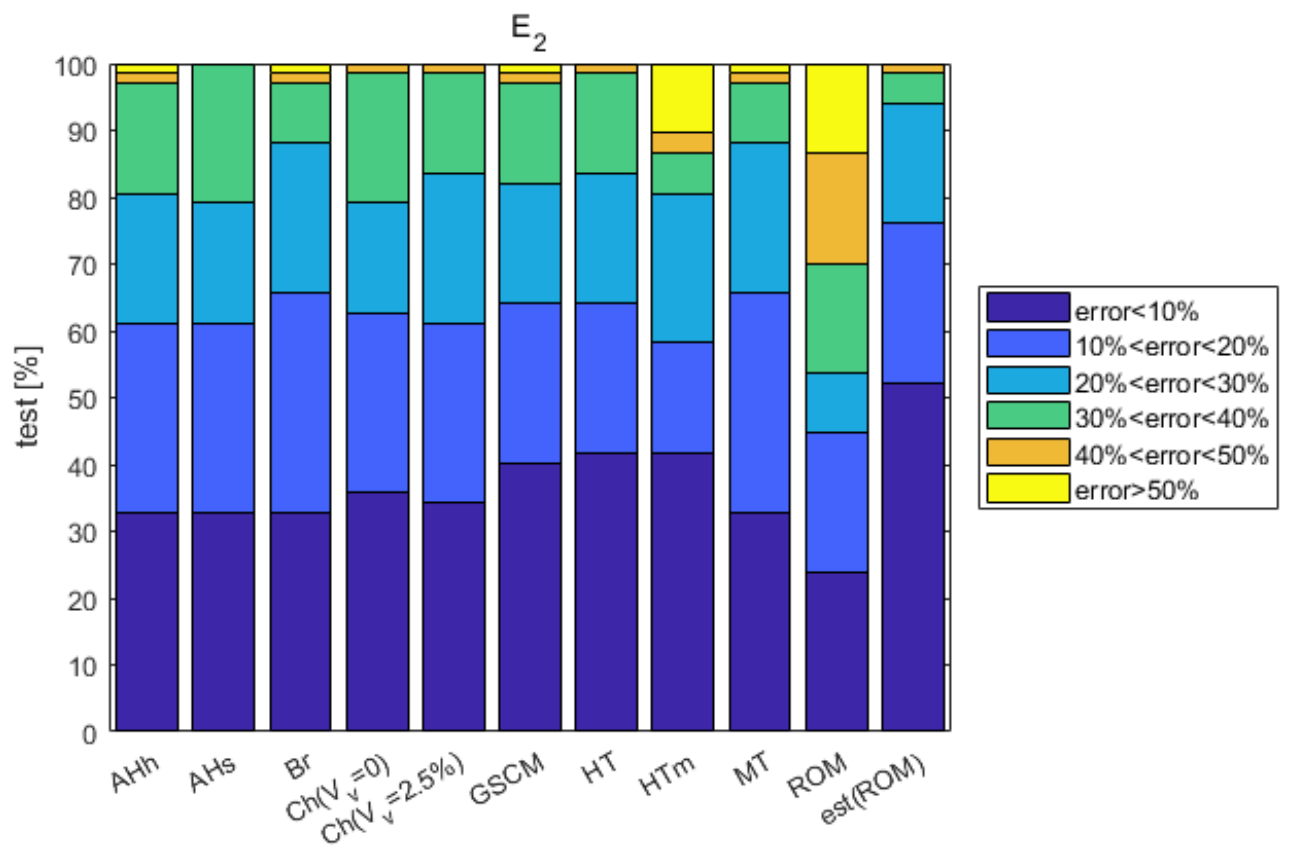

Figure 2: Ranges of error of the models estimations

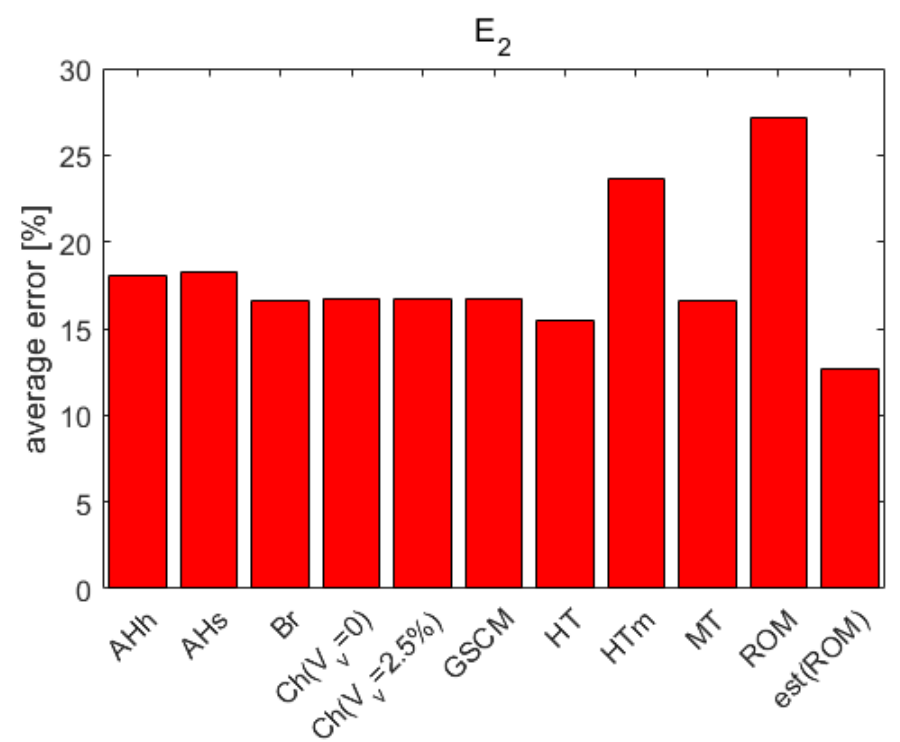

Figure 3: Average error of the models estimations

Several different manufacture processes were adopted for the compiled data. By this fact, a wide array of defects probably exists. For advanced manufactures, it is expected that the elasticity-based models become closer to the measured property, since none of them considers defects influence and the adjustable semi-empirical model considers this effect implicitly with calibrated terms. 


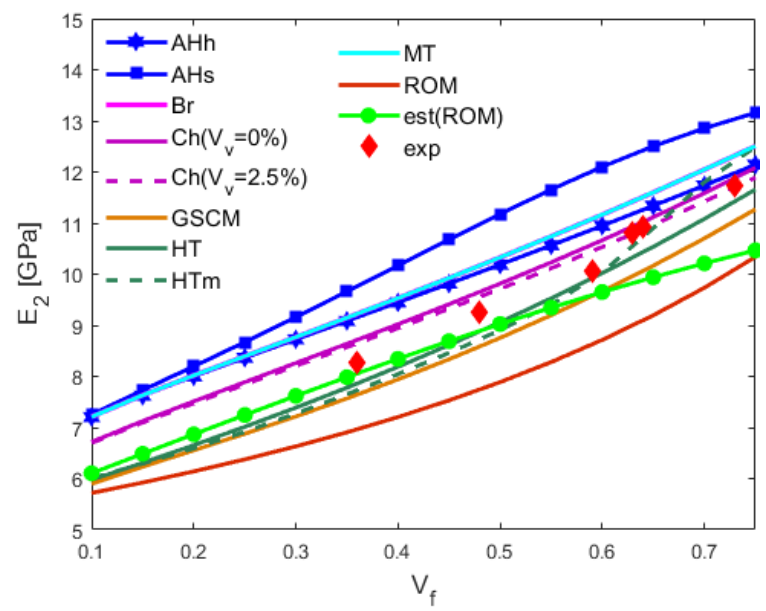

Figure 4: Comparision between models predictions and experimental data for carbon fiber $[20,25]$.
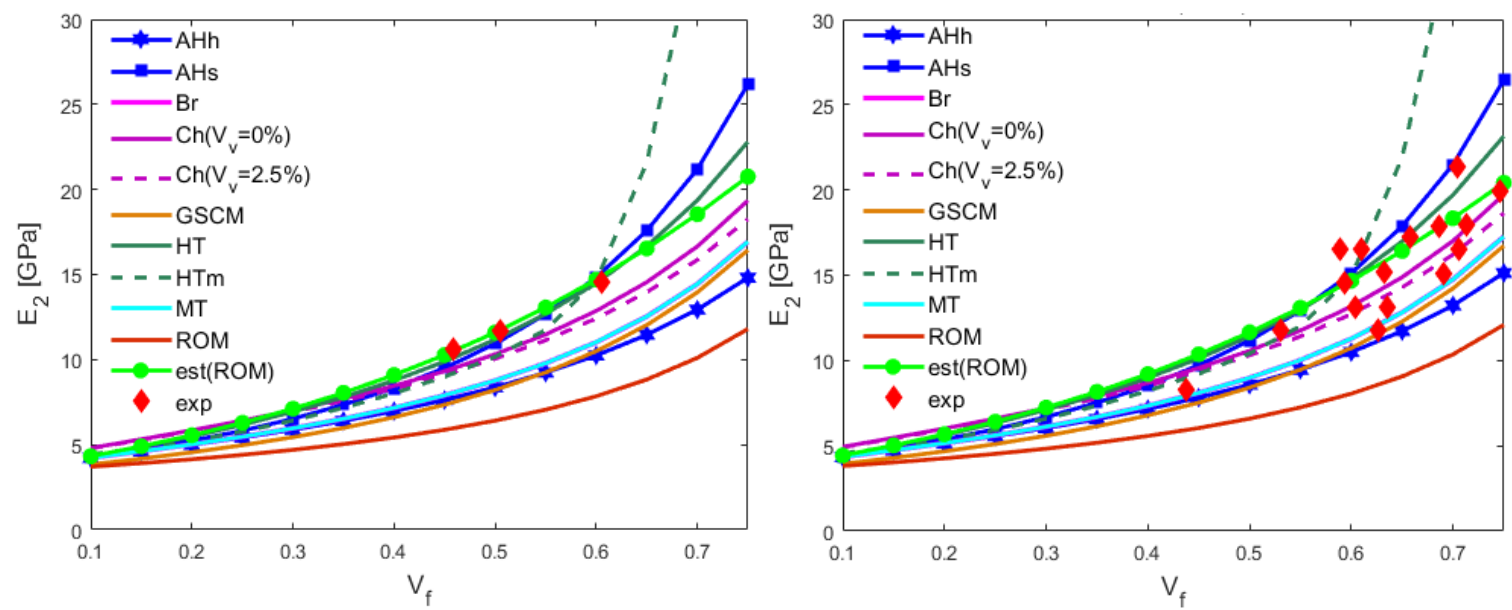

Figure 5: Comparision between models predictions and experimental data for glass fiber $[21,30]$.

To evaluate how $E_{2}$ varies according $V_{f}$, the models estimations are plotted together with the set of data presented by Kris \& Stinchcom [20] and Huang [25], Xin et al. [32] and Tsai \& Huhn [21] in Figures 4-5. The reference and the fiber type are quoted over the figures. The main conclusions are:

i) The modified Halpin-Tsai model has a good representation of the experimental data for carbon fibers, while for glass fibers this model tends to overestimate the transversal elastic modulus.

ii) The Bridging and Mori-Tanaka models have an estimation between the Asymptotic Homogenization with square and hexagonal symmetry.

iii) Based on the results of the Asymptotic Homogenization, the square symmetry results in a higher transversal modulus than the hexagonal.

iv) The proposed semi-empirical model is able to reproduce the experimental data with good precision.

\section{CONCLUSIONS}

This paper presents a comparative analysis of 10 different micromechanical models to estimate the transversal elastic modulus. The models predictions are compared with a set of 
65 experimental data compiled from literature. A semi-empirical model is proposed and a good correlation with experimental data is realized. This model obtained the smallest average error as well as the highest amount of case with error smaller than $10 \%$ (more than $50 \%$ of the cases).

\section{ACKNOWLEDGEMENTS}

The authors would like to acknowledge the support of the Brazilian Research Agencies CNPq, CAPES and FAPERJ. The Air Force Office of Scientific Research (AFOSR) is also acknowledged.

\section{REFERENCES}

[1] Soden, P.D., Kaddour, A.S., Hinton, M.J., 2004, "Recommendations for designers and researchers resulting from the world-wide failure exercise”, Compos. Sci. Technol., v. 64, pp. 589-604.

[2] Kaddour, A.S., Hinton, M.J., 2013, "Evaluation of theories for predicting failure in polymer composite laminates under 3-D states of stress", J Compos Mater, v. 47, pp. 6-7.

[3] Kaddour, A.S., Hinton, M.J., Smith, P.A., Li, S., 2013, "The background to the third worldwide failure exercise”, Journal of Composite Materials, v. 47, pp. 2417-2426.

[4] Selvadurai, A.P.S., Nikopour, H., 2012, "Transverse elasticity of a unidirectionally reinforced composite with an irregular fibre arrangement: Experiments, theory and computations", Composite Structures, v. 94, pp. 1973-1981.

[5] Barbero, E.J., 2018, Introduction to Composite Materials Design, 3 ed., CRC Press.

[6] Chamis, C.C., Abdi, F., Garg, M., Minnetyan, L., Baid, H., Huang, D., Housner, J., Talagani, F., 2013, "Micromechanics-based progressive failure analysis prediction for WWFE-III composite coupon test cases”, Journal of Composite Materials, v. 47, pp. 2695-2712.

[7] Halpin, J.C., Tsai, S.W., 1969, Effects of Environmental Factors on Composite Materials, AFML-TR-67-423.

[8] Halpin, J. C.; Kardos, J. L., 1976, “The Halpin-Tsai Equations: A Review”, Polymer Engineering and Science, v. 16, pp. 344-352.

[9] Ginet, E., Vercher, A., Marco, M., Arango, C., 2015, "Estimation of the reinforcement factor $n$ for calculating the transverse stiffness E2 with the Halpin-Tsai equations using the finite element method”. Composite Structures, v. 124, pp. 402-408.

[10] Nocedal, J., Wright, S.J., 1999, Numerical Optimization, Springer.

[11] Rodríguez-Ramos, R., Sabina, F.J., Guinovart-Díaz, R., Bravo-Castillero, J., 2001, "Closed-form expressions for the effective coefficients of a fiber-reinforced composite with transversely isotropic constituents - I. Elastic and square symmetry”, Mechanics of Materials, v. 33, pp. 223-235

[12] Guinovart-Díaz, R., Bravo-Castillero, J., Rodríguez-Ramos, R., Sabina, F.J., 2001, "Closed-form expressions for the effective coefficients of fibre-reinforced composite with transversely isotropic constituents. I: Elastic and hexagonal symmetry", Journal of the Mechanics and Physics of Solids, v. 49, pp. 1445-1462.

[13] Bravo-Castillero, J., Guinovart-Díaz, J., Rodríguez-Ramos, R., Sabina, F.J., Brenner, R., 2012, "Unified analytical formulae for the effective properties of periodic fibrous composites", Materials Letters, v. 73, pp. 68-71.

[14] Wang, Y.C., Huang, Z.M., 2015, “A new approach to a bridging tensor”. Polym. Compos., v. 36, pp. 1417-1431.

[15] Christensen, R., Miyano, Y., Nakada, M., 2015, "The size dependence of tensile strength for brittle isotropic materials and carbon fiber composite materials", Composites Science and Technology, v. 106, pp. 9-14.

[16] Mura, T., 1987, Micromechanics of defects in solids. Martinus Nijhoff.

[17] Benveniste, Y., 1987, “A new approach to the application of Mori-Tanaka's theory in composite materials”, Mechanics of Materials, v. 6, pp. 147-57. 
[18] Goggin, P.R., 1973, “The elastic constants of carbon-fibre composites”, Journal of Materials Science, v. 8, pp. 233-244.

[19] Holmes, M., Al-khayatt, Q.J., 1975, "Structural properties of grp”, Composites, v. 6, pp. 157-165.

[20] Kriz, R.D., Stinchcomb, W.W., 1979, "Elastic Moduli of Transversely Isotropic Graphite Fibers and Their Composites”, Experimental Mechanics, v. 19, pp. 41-49.

[21] Tsai, S.W., Hahn, H.T., 1980, Introduction to Composite Materials, Technomic.

[22] Wilczynsky, A.P., Lewinski, J., 1995, "Predicting the Properties of Unidirectional Fibrous Composites with Monotropic Reinforcement”, Composites Science and Technology, v. 55, pp. 139-143.

[23] Soden, P.D., Hinton, M.J., Kaddour, A.S., 1998, "Lamina Properties, Lay-up Configurations and Loading Conditions for a Range of Fibre-Reinforced Composite Laminates”, Composites Science and Technology, v. 58, pp. 1011-1022.

[24] Bledzki, A.K., Kessler, A., Rikard, R., Chate, A., 1999, "Determination of elastic constants of glass/epoxy unidirectional laminates by the vibration testing of plates", Composites Science and Technology, v. 59, pp. 2015-2024.

[25] Huang, H., Talreja, R., 2005, "Effects of void geometry on elastic properties of unidirectional fiber reinforced composites”, Composites Science and Technology, v. 65, pp. 1964-1981.

[26] Camanho, P.P., Miamí, P., Dávila, C.G., 2007, "Prediction of size effects in notched laminates using continuum damage mechanics”, Composites Science and Technology, v. 67, pp. 2715-2727.

[27] Kaddour, A.S., Hinton, M.J., 2012, "Input data for test cases used in benchmarking triaxial failure theories of composites", Journal of Composite Materials, v. 46, pp. 2295-2312.

[28] Kaddour, A.S., Hinton, M.J., Smith, P.A., Li, S., 2013, "Benchmarking of matrix cracking, damage and failure models for composites: comparison between theories". J Compos Mater 2013, v. 47, pp. 20-21.

[29] Schaefer, J.D., Werner, B.T., Daniel, I.M., 2014, "Strain-Rate-Dependent Failure of a Toughened Matrix Composite”, Experimental Mechanics, v. 54, pp. 1111-1120.

[30] Li, W., Cai, H., Zheng, J., 2014, "Characterization of Strength of Carbon Fiber Reinforced Polymer Composite Based on Micromechanics", Polymers and Polymer Composites, v. 22, pp. 105-116.

[31] Xin, H., Liu, Y., Mosallam, A.S., He, J., Du, A., 2017, "Evaluation on material behaviors of pultruded glass fiber reinforced polymer (GFRP) laminates", Composite Structures, v. 182, pp. 283-300. 